\title{
On the Numerical Solution of Convolution Integral Equations and Systems of such Equations
}

\author{
By J. G. Jones
}

1. Introduction. This paper discusses the application of a simple quadrature formula to the numerical solution of convolution integral equations of Volterra type and to systems of simultaneous equations of the same type. The convergence of the processes is considered in some detail, proofs being given that at a fixed value of the independent variable the errors in the solution tend to zero as the step length tends to zero.

2. Types of Equations Considered. The convolution integral equation to be solved is

$$
a g(x)-\int_{0}^{x} W(x-\xi) g(\xi) d \xi=f(x)
$$

where ' $a$ ' is a constant, and $f, W$ are given functions. The cases $a=0$ (equation of the first kind) and $a \neq 0$ (equation of the second kind) are discussed.

The corresponding system of equations can be written

$$
\mathbf{A g}(x)-\int_{0}^{x} \mathbf{W}(x-\xi) \mathbf{g}(\xi) d \xi=\mathbf{f}(x)
$$

where

$$
\begin{aligned}
& \mathrm{A}=\left[\begin{array}{ccc}
a_{11} & \cdots & a_{1 n} \\
\vdots & & \vdots \\
a_{n 1} & \cdots & a_{n n}
\end{array}\right], \text { a matrix of constants, } \\
& \mathbf{W}(z)=\left[\begin{array}{ccc}
W_{11}(z) & \cdots & W_{1 n}(z) \\
\vdots & & \vdots \\
W_{n 1}(z) & \cdots & W_{n n}(z)
\end{array}\right], \mathbf{f}(x)=\left[\begin{array}{c}
f_{1}(x) \\
\vdots \\
f_{n}(x)
\end{array}\right], \mathbf{g}(x)=\left[\begin{array}{c}
g_{1}(x) \\
\vdots \\
g_{n}(x)
\end{array}\right]
\end{aligned}
$$

and the notation involving integration is interpreted in an obvious way. For this system of equations the rank of $\mathbf{A}$ is an important parameter.

Equation (1) is well known, commonly occurring in practical problems. The system of equations (2) does not appear to have been previously discussed, so an instance of a practical problem in which it arises is briefly described.

In the linearized supersonic theory of [1] the system of equations (2) gives the pressure coefficients $f_{i}(x)$ on $n$ span wise wing stations produced by a quasi-cylindrical shaped fuselage defined by $n$ Fourier components $g_{i}(x)$. The $W_{i j}(z)$ are tabulated influence functions and $\mathbf{A}$ is of rank unity. In [2] the problem of determining the fuselage shape required to produce prescribed pressure coefficients on wing stations is considered. Special attention is there devoted to the numerical solution of (2) when $n$ equals 2 and the rank of $\mathbf{A}$ is unity.

Received March 31, 1959; revised November 16, 1959. 
3. The Use of the Trapezoidal Rule. In the method to be described, the equations are solved by dividing the $x$-axis into equal small intervals, replacing the integrals in the equations by the corresponding approximate expressions given by the trapezoidal rule, and hence deriving a formula for the step-by-step solution.

The solution of an integral equation by replacing the integral involved by a quadrature formula may be regarded as a standard method; see, for example, [3]. However, adequate discussions of the convergence of such numerical methods appear to be lacking. This paper is concerned with the convergence of the method in the particular case of the application of the trapezoidal rule to equation (1), and with the extension of the method to the solution of the system of equations (2).

The trapezoidal rule is the least accurate of quadrature formulas for a given step size, but it has the computational advantage of leading to a simple routine suitable for either desk or automatic computing, and furthermore is the most simple case to consider as regards convergence.

Subject to assumed bounds on the derivatives of the functions involved, it has been found possible to establish a bound for the truncation error at a given value of the independent variable, and hence to prove the convergence of the process.

The method of establishing a bound is not intended as a practical means of estimating errors. Practical ways of checking the accuracy of a numerical computation of this type are either to repeat the solution using a different step length, or to evaluate the integrals in the equations by using the numerical solution and a more accurate quadrature formula than the trapezoidal rule. However, it is shown in Section 8 that for equations of the first kind the numerical method described may in some cases also provide a convenient means of error analysis.

4. Sources of Error in the Solution. Consider the truncation error in this type of numerical solution. In going from the $m$ th step to the $(m+1)$ th, errors occur from two sources (neglecting rounding errors which it is assumed throughout are kept smaller than the truncation errors):

(i) due to replacing the integrals by a quadrature formula

(ii) due to the fact that the values of the solution at the first $m$ steps, all of which go into the quadrature formula for the evaluation of the solution at the $(m+1)$ th step, have corresponding errors in them.

In the following treatment the error at a fixed value of $x$ is considered and the step length is taken as an integral fraction of $x$. As the step length decreases, the decrease in the corresponding error produced in a single step is offset by the fact that the number of steps required to reach $x$ increases inversely. An analogous error analysis for the numerical solution of an ordinary differential equation is given in [4]. The form of the bound obtained in this latter case is similar to that of the bound described in Section 6 .

5. The Single Integral Equation. In this section we consider the numerical solution of (1). If $a \neq 0$ the step-by-step process is started by means of the equation

$$
a g(0)=f(0) .
$$

If $a=0$, (1) gives, on differentiation, 


$$
-W(0) g(x)-\int_{0}^{x} W^{\prime}(x-\xi) g(\xi) d \xi=f^{\prime}(x) .
$$

If now $W(0) \neq 0$, the step-hy-step process is started hy means of the equation

$$
-W(0) g(0)=f^{\prime}(0) \text {. }
$$

If $W(0)=0$, further differentiation $(n+1)$ times is required until we reach a derivative $W^{(n)}(0) \neq 0$. have

Equal intervals of length $\delta$ are now taken along the $x$-axis. For the $m$ th step we

$$
a g(m \delta)-\int_{0}^{m \delta} W(m \delta-\xi) g(\xi) d \xi=f(m \delta) .
$$

Replacing the integral hy the corresponding trapezoidal rule approximation and rearranging:

$$
\begin{aligned}
\left\{a-\frac{\delta}{2} W(0)\right\} g^{*}(m \delta) & =f(m \delta) \\
& +\delta\left\{\frac{1}{2} W(m \delta) g(0)+\sum_{i=1}^{m-1} W([m-i] \delta) g^{*}(i \delta)\right\}
\end{aligned}
$$

where an asterisk denotes an approximate value (and we use the convention throughout that $\sum_{i=1}^{0} \equiv 0$ ).

tinlo; both $a=0$ and $W(0)=0$ we can successively evaluate

$$
g^{*}(\delta), g^{*}(2 \delta), \cdots \text {. }
$$

If $a=0$ and $W(0)=0$, then the equivalent equation (1) (or if necessary an equation obtained hy further differentiation) is solved by the method described. The additional errors involved in numerical differentiation (assuming the functions to have been given in tabulated form) are discussed in Section 8.

The equation corresponding to (6) involving the true values is

$$
\begin{aligned}
\left\{a-\frac{\delta}{2} W(0)\right\} g(m \delta) & =f(m \delta) \\
+ & \delta\left\{\frac{1}{2} W(m \delta) g(0)+\sum_{i=1}^{m-1} W([m-i] \delta) g(i \delta)\right\}+\sum_{i=1}^{m} e_{m, i}
\end{aligned}
$$

where

$$
\begin{aligned}
e_{m, i}=\int_{(i-1) \delta}^{i \delta} W(m \delta-\xi) g(\xi) d \xi \\
-\frac{\delta}{2}\{W(m \delta-[i-1] \delta) g([i-1] \delta)+W(m \delta-i \delta) g(i \delta)\} .
\end{aligned}
$$

Writing

$$
E_{m}=g^{*}(m \delta)-g(m \delta)
$$


(i.e., $E_{m}$ is the error after $m$ steps) equations (6) and (7) give

$$
\left\{a-\frac{\delta}{2} W(0)\right\} E_{m}=\delta \sum_{i=1}^{m-1} W([m-i] \delta) E_{i}-\sum_{i=1}^{m} e_{m, i}
$$

Now (see, for example, [5]) from (8)

$$
e_{m, i}=-\frac{1}{12} \delta^{2}\left\{\frac{d}{d \xi}[W(m \delta-\xi) g(\xi)]_{\xi-i \delta}-\frac{d}{d \xi}[W(m \delta-\xi) g(\xi)]_{\xi-(i-1) \delta}\right\}+O\left(\delta^{5}\right) \text {. }
$$

If we assume that $g$ and $W$ have bounded derivatives up to the second, it follows that $e_{m, i}$ is $O\left(\delta^{3}\right)$.

From equation (10) it follows by induction that a positive constant $K$ can be found such that

$$
\left|E_{m}\right| \leqq f_{m}
$$

where

$$
\left\{a-\frac{\delta}{2} W(0)\right\} f_{m}=K \delta \sum_{i=1}^{m-1} f_{i}+m K \delta^{3} .
$$

Rewriting (11) with $(m+1)$ for $m$ and subtracting (11) from the result,

$$
\left\{a-\frac{\delta}{2} W(0)\right\} f_{m+1}-\left\{a-\frac{\delta}{2} W(0)+\delta K\right\} f_{m}=K \delta^{3}
$$

The solution of this difference equation with initial condition from (11) is

$$
f_{m}=C_{1}\left\{\frac{a-\frac{\delta}{2} W(0)+\delta K}{a-\frac{\delta}{2} W(0)}\right\}^{m}-\delta^{2}
$$

where

$$
C_{1}=\frac{K \delta^{3}+\delta^{2}\left(a-\frac{\delta}{2} W(0)\right)}{a-\frac{\delta}{2} W(0)+\delta K}
$$

The two cases $a \neq 0$ and $a=0$ are discussed separately in the next two sections.

6. Equation of the Second Kind $(a \neq 0)$. In this case (12) provides the required bound. It is similar to the bound obtained for the error in the numerical solution of an ordinary differential equation in [4].

At a fixed value of $x$ we consider a sequence of values of $\delta$ such that $m \delta=x$ and $m \rightarrow \infty$ as $\delta \rightarrow 0$. Then for small $\delta$ the term in (12) with index $m$ is bounded and $C_{1}=0\left(\delta^{2}\right)$. So for a fixed value of $x, f_{m}=0\left(\delta^{2}\right)$, hence $E_{m}=O\left(\delta^{2}\right)$.

By considering the particular case $W=$ constant $=K>0$ and $g^{\prime \prime}=$ constant $=$ 12 it can be seen that the error $E_{m}$ can attain its bound $f_{m}$, and so, for given step size, it is possible for $E_{m}$ to increase with $m$ like $A^{m}$, where $A>1$. 
7. Equation of the First Kind $(a=0)$. We assume in this section that $W(0) \neq 0$, in which case equation (1), with $a=0$, can be solved directly by means of the numerical formula (6).

In this case equation (12) only provides a convergent bound for $E_{m}$ (at a fixed value of $x$ ) if

$$
\left|1-\frac{2 K}{W(0)}\right| \leqq 1
$$

In general $K$ cannot be chosen to satisfy this condition. An example of an equation for which (12) does provide a bound is

$$
\int_{0}^{x} g(\xi) d \xi=2 x^{3}
$$

in which $W=$ constant $=1$. In this case it is easily shown that the errors satisfy the equation

$$
-\frac{1}{2} E_{m}=\sum_{i=1}^{m-1} E_{i}+m \delta^{2}
$$

On comparison with (11) it can be seen that we can choose $K=1$ in order to satisfy the equality $E_{m}=f_{m}$. In this case (13) is satisfied, and as in Section 6 it follows that for a fixed value of $x, E_{m}=O\left(\delta^{2}\right)$. A point of interest is that the errors $E_{m}$ in this example form an oscillating sequence, viz., $-2 \delta^{2}, 0,-2 \delta^{2}, 0, \cdots$. This behavior is typical of the errors when $a=0$, as is shown by the following analysis, which also shows that a convergent bound can always be found.

Equation (10) in the present case reduces to

$$
-\frac{\delta}{2} W(0) E_{m}=\delta \sum_{i=1}^{m-1} W([m-i] \delta) E_{i}-\sum_{i=1}^{m} e_{m, i}
$$

So

$$
-\frac{\delta}{2} W(0)\left\{E_{m+1}-E_{m}\right\}=\delta W(\delta) E_{m}+J_{m}
$$

where

$$
\begin{aligned}
J_{m}=\delta \sum_{i=1}^{m-1}\{W([m+1-i] \delta)-W([m-i] \delta)\} E_{i} & \\
& -e_{m+1, m+1}-\sum_{i=1}^{m}\left(e_{m+1, i}-e_{m, i}\right) .
\end{aligned}
$$

Equation (17) can be rearranged to give

$$
-\frac{\delta}{2} W(0)\left\{E_{m+1}+E_{m}\right\}=\delta\{W(\delta)-W(0)\} E_{m}+J_{m} .
$$

The right hand side of (19) is in general smaller than the right hand side of (17). That is, $E_{m+1}+E_{m}$ is in general smaller than $E_{m+1}-E_{m}$, which implies that $E_{m+1}$ and $E_{m}$ are in general of opposite sign. The $E_{m}$ then forms an oscillating 
sequence. A smaller bound than that given by equation (12), which is obtained essentially from (10) by replacing the errors by their moduli, can therefore be obtained by relating $E_{m+2}$ to $E_{m}$.

Replacing $m$ by $m+1$ in (19) and subtracting (19) as it stands gives an equation for $E_{m+2}-E_{m}$. Assuming that $g$ and $W$ have bounded derivatives up to fourth order, it follows that the first and second differences of $W$ are respectively of orders $\delta$ and $\delta^{2}$ and that the first and second differences of $e_{m, i}$ are respectively of orders $\delta^{4}$ and $\delta^{5}$. It can then be shown inductively that a positive constant $K$ can be found such that

$$
\left|E_{m}\right| \leq h_{m}
$$

where

$$
h_{m+2}-h_{m}=K\left\{\delta^{2} \sum_{i=1}^{m-1} h_{i}+\delta\left(h_{m+1}+h_{m}\right)+m \delta^{4}+\delta^{3}\right\},
$$

and $h_{1}, h_{2}$ are chosen so that (20) holds for $m=1,2$.

From (16) it can be seen that $h_{1}$ and $h_{2}$ can be chosen so that

$$
h_{1}=O\left(\delta^{2}\right), \quad h_{2}=O\left(\delta^{2}\right)
$$

and

$$
h_{1}<h_{2} \text {. }
$$

Then

$$
h_{2 m-1}<h_{2 m} \text {, all } m
$$

and hence it may be verified by induction that if

$$
H_{2 m+2}-H_{2 m}=K\left\{\delta^{2}\left(2 \sum_{i=1}^{m-1} H_{2 i}+H_{2 m}\right)+\delta\left(H_{2 m}+H_{2 m+2}\right)+2 m \delta^{4}+\delta^{3}\right\}
$$

and

$$
H_{2}=h_{2}
$$

then

$$
h_{2 m}<H_{2 m} \text { all } m>1 .
$$

From (25) there is obtained the difference equation

$$
H_{2 m+4}(1-K \delta)-H_{2 m+2}\left(2+K \delta^{2}\right)+H_{2 m}\left(1-K \delta^{2}+K \delta\right)=2 K \delta^{4} .
$$

The solution of $(28)$ is

$$
H_{2 m}=-\delta^{2}+C_{1} p_{1}{ }^{m}+C_{2} p_{2}{ }^{m}
$$

where $p_{1}, p_{2}$ are the two values of

$$
\frac{1}{1-K \delta}\left\{1+\frac{K \delta^{2}}{2} \pm\left[K(2+K) \delta^{2}-K^{2} \delta^{3}+\frac{K^{2} \delta^{4}}{4}\right]^{1 / 2}\right\}
$$

and $C_{1}, C_{2}$ are given by the initial conditions

$$
\left\{\begin{array}{l}
p_{1} C_{1}+p_{2} C_{2}=H_{2}+\delta^{2} \\
p_{1}^{2} C_{1}+p_{2}^{2} C_{2}=H_{4}+\delta^{2}
\end{array}\right.
$$


Now from (22) and (26), $H_{2}=O\left(\delta^{2}\right)$ and from (25), $H_{4}=H_{2}(1+O(\delta))$. Also from (30), $p_{1}=1+O(\delta), p_{2}=1+O(\delta), p_{2}-p_{1}=O(\delta)$. So from (31),

$$
C_{1}=\frac{p_{2}\left(H_{2}+\delta^{2}\right)-\left(H_{4}+\delta^{2}\right)}{p_{1}\left(p_{2}-p_{1}\right)}=\frac{O\left(\delta^{3}\right)}{O(\delta)}=O\left(\delta^{2}\right) \text {. }
$$

Similarly $C_{2}=O\left(\delta^{2}\right)$.

At a fixed value of $x$ we consider a sequence of values of $\delta$ such that $2 m \delta=x$, thus $m \rightarrow \infty$ as $\delta \rightarrow 0$. Then for small $\delta$ the terms in (29) with index $m$ are bounded and $C_{1}, C_{2}$ are $O\left(\delta^{2}\right)$. So for fixed $x, H_{2 m}$ is $O\left(\delta^{2}\right)$ and from (27), (24) and (20) it follows that $E_{m}=O\left(\delta^{2}\right)$.

8. Comparison of Methods. An equation of the first kind ( $a=0)$ can be converted into an equation of the second kind by differentiation. For example, if $W(0) \neq 0$ equation (4) results. It has already been shown in Sections 6 and 7 that for both types of equation the truncation error at a given value of the independent variable is $O\left(\delta^{2}\right)$. In this section the effects of rounding errors in the two cases are briefly considered and the problem of whether to convert a first order equation into a second order one by differentiation before solving numerically is discussed.

In the case $a=0$ the numerical formula (6) becomes

$$
g^{*}(m \delta)=\frac{2}{W(0)}\left\{-\frac{f(m \delta)}{\delta}-\left[\frac{1}{2} W(m \delta) g(0)+\sum_{i=1}^{m-1} W([m-i] \delta) g^{*}(i \delta)\right]\right\} .
$$

In the first place, as was shown in Section 7 , the truncation error in $g^{*}(m \delta)$ obtained by using the formula (32) is in general of opposite sign for two consecutive values of $m$. Because of this the truncation error in the summation in the right hand side of (32) and hence in $g^{*}(m \delta)$ is smaller than it would be if the errors were of the same sign. To take advantage of this in the numerical computation, several digits must be retained beyond the point where the truncation error begins before rounding off. This implies that $W$ must be known to several more significant figures than would otherwise be necessary. It is also evident on comparison of (6) and (32) that in the latter formula more digits in $f(m \delta)$ must be retained before rounding because of the $\delta$ in the denominator.

Suppose now that we are to solve an equation of the first kind, viz. (1) with $a=0$. The functions $f$ and $W$ are supposed to have been given in tabular form and $W(0) \neq 0$. Then the equation can be solved directly or it can be differentiated first, giving (4), an equation of the second kind.

If the first method is adopted, then, as has been shown, the truncation errors will in general be of opposite sign and the rounding errors must be kept several digits smaller than the truncation error. If an automatic computer is being used it may not be inconvenient to retain these extra significant figures. However, $f$ and $W$ must be known to the extra degree of accuracy. Since the truncation errors are in general of opposite sign a simple smoothing process may be employed to improve the solution as follows. Denote the sequence of numbers

$$
g(0), g^{*}(2 \delta), g^{*}(4 \delta), \cdots
$$

by the symbols

$$
g_{e}(0), g_{e}(2 \delta), g_{e}(4 \delta), \cdots
$$


and complete the sequence

$$
g_{e}(0), g_{e}(\delta), g_{c}(2 \delta), \cdots
$$

by interpolation.

Similarly, from the sequence of numbers

$$
g^{*}(\delta), g^{*}(3 \delta), g^{*}(5 \delta), \cdots
$$

the sequence

$$
g_{0}(\delta), g_{0}(2 \delta), g_{0}(3 \delta), \cdots
$$

$\mathbf{i}_{\mathrm{S}}$ formed by using interpolation.

The two sequences $g_{e}(m \delta), g_{0}(m \delta)$ then give approximate bounds to the solution, and the smoothed solution is given by $g_{s}(m \delta)=\frac{1}{2}\left[g_{e}(m \delta)+g_{0}(m \delta)\right]$. This procedure is illustrated in the numerical computation at the end of this section (Table 1).

If the second method is adopted, that is, equation (4) is solved instead, the functions $f$ and $W$ must first be differentiated numerically. Once this has been done the numerical solution can be rounded off to the same degree of accuracy as the truncation error and consequently fewer significant figures need be retained. However, extra significant figures in $f$ and $W$ now have to be used in the first place to obtain $f^{\prime}$ and $W^{\prime}$ to the required degree of accuracy when using numerical differentiation.

Each method appears to have its advantages, and the choice must depend on the data provided in a given problem, that is, on the spacing and number of significant figures in the given functions.

We conclude this section by presenting the details of a simple numerical computation in which the results given by the two methods can be compared.

The equation is

$$
\int_{0}^{x} W(x-\xi) g(\xi) d \xi=f(x)
$$

where $W(x)=\cos x$ and $f(x)=\sin x$. The analytical solution is $g(\xi)=1$. The functions are given to 4 significant figures at intervals of 0.1 in $x$. The functions are differentiated numerically using a three-point formula except for the values at

\begin{tabular}{|c|c|c|c|c|c|c|c|c|c|}
\hline$x$ & $\begin{array}{l}W^{\prime}(x) \\
(=\cos x)\end{array}$ & $\begin{array}{c}f(x) \\
(=\sin x)\end{array}$ & $\begin{array}{c}g^{*}(x) \\
\text { (direct } \\
\text { solution) }\end{array}$ & $\operatorname{se}_{e}(x)$ & $g_{0}(x)$ & $g_{8}(x)$ & $\begin{array}{c}W^{\prime}(x) \\
\text { (3-point } \\
\text { numerical } \\
\text { formula) }\end{array}$ & $\begin{array}{c}f^{\prime}(x) \\
\text { (3-point } \\
\text { formula) }\end{array}$ & $\begin{array}{c}f^{*}(x) \\
\text { (differentiated } \\
\text { equation) }\end{array}$ \\
\hline $\begin{array}{l}0 \\
0.1 \\
0.2 \\
0.3 \\
0.4 \\
0.5 \\
0.6 \\
0.7 \\
0.8 \\
0.9 \\
1.0\end{array}$ & $\begin{array}{l}1.0000 \\
0.9950 \\
0.9800 \\
0.9554 \\
0.9211 \\
0.8776 \\
0.8253 \\
0.7649 \\
0.6968 \\
0.6216 \\
0.5402\end{array}$ & $\begin{array}{l}0 \\
0.0999 \\
0.1988 \\
0.2954 \\
0.3894 \\
0.4795 \\
0.5647 \\
0.6441 \\
0.7173 \\
0.7833 \\
0.8415\end{array}$ & $\begin{array}{l}1.000 \\
1.003 \\
1.000 \\
0.997 \\
1.006 \\
0.998 \\
1.003 \\
0.995 \\
1.008 \\
0.994 \\
1.009\end{array}$ & $\begin{array}{l}1.000 \\
1.000 \\
1.000 \\
1.003 \\
1.006 \\
1.004 \\
1.003 \\
1.006 \\
1.008 \\
1.008 \\
1.009\end{array}$ & $\begin{array}{l}1.000 \\
1.003 \\
1.000 \\
0.997 \\
0.998 \\
0.998 \\
0.996 \\
0.995 \\
0.994 \\
0.994\end{array}$ & $\begin{array}{l}1.000 \\
1.002 \\
1.000 \\
1.000 \\
1.002 \\
1.001 \\
1.000 \\
1.000 \\
1.001 \\
1.001\end{array}$ & $\begin{array}{c}0 \\
-0.100 \\
-0.198 \\
-0.294 \\
-0.389 \\
-0.479 \\
-0.564 \\
-0.642 \\
-0.716 \\
-0.783\end{array}$ & $\begin{array}{l}1.000 \\
0.994 \\
0.978 \\
0.953 \\
0.920 \\
0.876 \\
0.823 \\
0.763 \\
0.696 \\
0.621\end{array}$ & $\begin{array}{l}1.000 \\
0.999 \\
0.998 \\
0.997 \\
0.998 \\
0.998 \\
0.997 \\
0.997 \\
0.998 \\
0.998\end{array}$ \\
\hline
\end{tabular}
$x=0$ where a four-point formula is used. The interpolation in the formation of the sequences $g_{e}(m \delta), g_{0}(m \delta)$ is linear.

TABLE 1 
9. The System of Integral Equations. In this section we consider the numerical solution of the system (2). We use the expression $r(\boldsymbol{A})$ to denote the rank of $A$.

If $r(A)=n$, the step-by-step process is started by solving the algebraic set of equations

$$
\operatorname{Ag}(0)=f(0) .
$$

If $r(A)<n$ then $n$ linear combinations of the equations (2) can be chosen to give another system of the same form, with a matrix in which $n-r$ rows are identically zero. If the $n-r$ corresponding equations are differentiated, the rank of the matrix of the resulting set of $n$ equations will in general have increased (in the same way that differentiation of the equation of the first kind, viz. (1) with $a=0$, in general gives equation (4) of the second kind). We assume that repeated application of this process eventually leads to a system of the same form as (2) with a matrix of rank $n$ (this corresponds to the assumption in Section $5: W^{(n)}(0) \neq 0$ for some $n$ ). Setting $x=0$ in this new system, we obtain a set of equations analogous to (33) with which to start the step-by-step process.

Equal intervals of length $\delta$ are now taken along the $x$-axis. For the $m$ th step we have

$$
\mathbf{A g}(m \delta)-\int_{0}^{m \delta} \mathbf{W}(m \delta-\xi) \mathbf{g}(\xi) d \xi=\mathbf{f}(m \delta) .
$$

Replacing the integral by the corresponding trapezoidal rule approximation and rearranging:

$$
\begin{aligned}
\left\{\mathbf{A}-\frac{\delta}{2} \mathbf{W}(0)\right\} \mathbf{g}^{*}(m \delta)= & \mathbf{f}(m \delta) \\
& \quad+\delta\left\{\frac{1}{2} \mathbf{W}(m \delta) \mathbf{g}(0)+\sum_{i=1}^{m-1} \mathbf{W}([m-i] \delta) \mathbf{g}^{*}(i \delta)\right\} \\
= & Q(m \delta) \quad(\text { say }),
\end{aligned}
$$

where an asterisk denotes an approximate value.

Provided $\left|A-\frac{\delta}{2} \mathbf{W}(0)\right| \neq 0$ we can successively evaluate

$$
\mathbf{g}^{*}(\boldsymbol{\delta}), \mathbf{g}^{*}(2 \delta), \cdots
$$

where at each step we solve a set of $n$ equations (with coefficients independent of $n$ ).

The above method is exactly analogous to that described for the single integral equation in Section $\boldsymbol{\jmath}$. Only the outlines of proofs of convergence are given in this section, the details being analogous to those given for the single integral equation. The equation for the truncation error corresponding to (10) is

$$
\left\{\mathbf{A}-\frac{\delta}{2} \mathbf{W}(0)\right\} \mathbf{E}_{m}=\delta \sum_{i=1}^{m-1} \mathbf{W}([m-i] \delta) \mathbf{E}_{i}-\sum_{i=1}^{m} \mathbf{e}_{m, i}
$$

where

$$
E_{m}=g^{*}(m \delta)-\mathbf{g}(m \delta)
$$


and

$$
\begin{aligned}
& \epsilon_{m, i}=\int_{(i-1) \delta}^{i \delta} \mathbf{W}(m \delta-\xi) g(\xi) d \xi \\
&-\frac{\delta}{2}\{\mathbf{W}(m \delta-[i-1] \delta) g([i-1] \delta)+W(m \delta-i \delta) g(i \delta)\} .
\end{aligned}
$$

The case of $r(A)=n$ corresponds to the case $a \neq 0$ in Section 5 and a quantity $f_{m}$ can be found which is a bound for every component of $\mathbf{E}_{m}$ and is of the same form as (12) (with $a \neq 0$ ). So when $r(A)=n$, at a fixed value of $x$ we have $E_{m}=$ $O\left(\delta^{2}\right)$. In this case there is no condition on $r(W(0))$.

If $r(A)<n$ the analysis proceeds as in Section 7. In this case we assume, to avoid further complication, $r(W(0))=n$. The equation corresponding to (17) is

$$
\left\{A-\frac{\delta}{2} \mathbf{W}(0)\right\}\left\{\mathbf{E}_{m+1}-\mathbf{E}_{m}\right\}=\delta \mathbf{W}(\delta) \mathbf{E}_{m}+J_{m}
$$

where

$$
\begin{aligned}
J_{m}=\delta \sum_{i=1}^{m-1}\{\mathbf{W}([m+1-i] \delta)-W([m-i] \delta)\} \mathbf{E}_{i} \\
\\
\quad-\mathbf{e}_{m+1, m+1}-\sum_{i=1}^{m}\left(\mathbf{e}_{m+1, i}-\mathbf{e}_{m, i}\right) .
\end{aligned}
$$

Equation (36) can be rearranged to give

$$
\left\{A-\frac{\delta}{2} \mathbf{W}(0)\right\}\left\{\mathbf{E}_{m+1}+\mathbf{E}_{m}\right\}=2 \mathbf{A} \mathbf{E}_{m}+\delta\{\mathbf{W}(\delta)-\mathbf{W}(0)\} \mathbf{E}_{m}+J_{m} .
$$

This is of the same form as equation (19) except for the term $2 \mathrm{AE}_{\mathrm{m}}$. It is now shown that when $r(A)<n$ this term only contributes terms to the elements of $\mathbf{E}_{m+1}+\mathbf{E}_{m}$ of the same order as the contribution of the remaining terms on the right hand side of (38). We assume that the elements of the last $n-r$ rows of $\dot{A}$ are all zero (this can be arranged by taking linear combinations of the original equations). Then

$$
\left|A-\frac{\delta}{2} W(0)\right|=O\left(\delta^{n \rightarrow}\right),
$$

and it is easily verified that each element of $\mathbf{E}_{m+1}+\mathbf{E}_{m}$ consists of a linear combination of the elements of the right hand side of (38), the coefficients of the first $r$ elements being $O(1)$ and those of the last $n-r$ elements being $O\left(\delta^{-1}\right)$. But all the last $n-r$ elements of $\mathbf{A \mathbf { E } _ { m }}$ vanish, so the dominant terms in each element of $\mathbf{E}_{m+1}+$ $\mathbf{E}_{m}$ are of the type

(a) $O\left(\delta^{-1}\right)\left\{\delta[\mathbf{W}(\delta)-W(0)] E_{m}+J_{m}\right\}_{i}$

$$
\text { where } r+1 \leqq i \leqq n \text {, }
$$

(b) $O$ (1) $\left\{\mathbf{A E}_{m}\right\}_{\text {i }}$

$$
\text { where } 1 \leqq i \leqq r .
$$

The term (a) is analogous to the expression for $E_{m+1}+E_{m}$ from equation (19). 
From equation (3.5)

$$
\mathbf{A} \mathbf{E}_{m}=\frac{\delta}{2} \mathbf{W}(0) \mathbf{E}_{m}+\delta \sum_{i=1}^{m-1} \mathbf{W}([m-i] \delta) \mathbf{E}_{i}-\sum_{i=1}^{m} \mathbf{e}_{m, i} .
$$

It can be seen that contributions from (a) and (b) are of the same order.

The situation is the same as in Section 7, the elements of $\mathbf{E}_{m+1}+\mathbf{E}_{m}$ being in general smaller than those of $\mathbf{E}_{m+1}-\mathbf{E}_{m}$, consecutive errors thus being in general of opposite sign. Replacing $m$ by $m+1$ in (38) and subtracting (38) as it stands gives an equation for $\mathbf{E}_{m+2}-\mathbf{E}_{m}$. Assuming that the elements of $\mathbf{g}$ and $\mathbf{w}$ have bounded derivatives up to the fourth order it can be shown inductively that a positive constant $K$ can be found such that

$$
\left|\left\{\mathbf{E}_{m}\right\}_{i}\right| \leqq h_{m}, \text { for all } i
$$

where $h_{m}$ is given by (21), and (22), (23) hold. The analysis of Section 7 then shows that for a fixed value of $x$ we have $E_{m}=O\left(\delta^{2}\right)$.

As indicated at the beginning of this section, if $r(\mathbb{A})<n$ the system can in general be converted into an equivalent system with $r=n$ by application of the operations of addition and differentiation. The problem of whether to convert a system with $r<n$ into an equivalent system in this way, before solving numerically, is exactly analogous to that discussed in Section 8, and will not be treated further here. The merits of the two methods are summarized in the conclusions.

10. Conclusions. The numerical solution of a convolution integral equation of the Volterra type, equation (1), by using a simple quadrature formula has been discussed. It is shown that if the step length is $\delta$, the truncation error at a fixed value of the independent variable is $O\left(\delta^{2}\right)$ both for equations of the first and second kinds. However, the behavior of the truncation error is different in the two cases, being in general of opposite sign for consecutive steps in the case of an equation of the first kind. Since an equation of the first kind can in general be converted into one of the second kind by differentiation, it can either be solved numerically as it stands or differentiated first. The merits of the two methods appear to be as follows. In the direct method, both a smooth solution and approximate bounds for the truncation error can be obtained simultaneously. However, more significant figures have to be retained throughout before rounding off. If the equation is differentiated first, the given functions (which are assumed to be given in tabulated form) have to be differentiated numerically. In the actual step-by-step computation fewer significant figures need be retained. If the given functions are tabulated to sufficient significant figures to permit accurate derivatives to be obtained using numerical differentiation, the second method will probably be the more accurate. Otherwise, and if it is convenient to retain the extra significant figures throughout, the direct method has the advantages of requiring fewer steps (no numerical differentiation) and of providing approximate bounds for the truncation error. The method has been generalized to include the numerical solution of the system of integral equations (2). An instance of a practical problem arising from supersonic linearized theory in which this equation occurs has been described. The truncation error at a fixed value of the independent variable is again $O\left(\delta^{2}\right)$. The behavior of the truncation error is analogous to that in the solution of the single integral equation, the case $r(\boldsymbol{A})<n$ 
corresponding to an equation of the first kind, and the case $r(\mathbf{A})=n$ corresponding to an equation of the second kind.

11. Acknowledgement. The author is indebted to the referee for some helpful suggestions.

Royal Aircraft Establishment

Bedford, England.

1. J. N. Nielsen \& W. C. PitTs, "General theory of wave-drag reduction for combinations emploving quasi-cylindrical bodies with an application to swept wing and body combinations," NACA TN 3722, National Advisory Committee for Aeronautics, Washington, D. C., September 1956.

2. J. G. JoNEs, "A method for designing body shape to produce prescribed pressure distributions on wing-body combinations at supersonic speeds," (to be published as (British) ARC Current Paper).

3. L. Fox \& E. T. Goodwr, "The numerical solution of non-singular linear integral equations," Philos. Trans. Roy. Soc. London, Ser. A, v. 245, February 1953.

4. W. E. MIrL.se, Numerical Solution of Differential Equations, John Wiley \& Sons, Inc., New York, 1953.

5. D. R. Hartree, Numerical Analysis, Oxford University Press, London, 1952. 